\title{
AVALIAÇÃO QUALITATIVA E QUANTITATIVA DO ESVAZIAMENTO GÁSTRICO ATRAVÉS DO MÉTODO VIDEOFLUOROSCÓPICO+
}

\author{
Claudia M. G. FIRMAN*, Milton M. B. COSTA**, Manoel L. COSTA** e Eponina LEMME***
}

RESUMO - Estudo do esvaziamento gástrico de 21 voluntários sadios, usando dieta padrão composta por homogeneizado de lipídios, carboidratos, proteínas e solução de sulfato de bário. Efetuaram-se, para cada voluntário, seis registros videofluoroscópicos em incidência póstero-anterior, com duração de um minuto, a intervalos de 12 minutos na primeira hora e, a seguir, a cada 30 minutos, até o esvaziamento total. Oito exames foram selecionados para a quantificação do esvaziamento. A análise da seqüência de registros videofluoroscópicos permitiu observar e estimar, de modo subjetivo, o comportamento funcional e o ritmo do esvaziamento gástrico. A digitalização de imagens representativas dos diversos tempos do esvaziamento gástrico permitiu quantificar as observações e estabelecer uma curva índice deste. A quantificação foi realizada com o uso de rotina desenvolvida no software Image pro-plus da Media Cybernetics. Conclusões - 1. A videofluoroscopia permitiu, com adequada resolução, a análise qualitativa da motilidade e do esvaziamento gástrico. 2. É possivel quantificar as variações de forma que se observa na seqüência de imagens registradas pelo método videofluoroscópico. 3. Os resultados expressam um índice de esvaziamento gástrico, determinado pela variação temporal da projeção da área gástrica em imagens radiológicas obtidas em incidência póstero-anterior. 4. A análise quantitativa não deve prescindir da análise qualitativa.

DESCRITORES - Fluoroscopia. Motilidade gástrica. Esvaziamento gástrico.

\section{INTRODUÇÃO}

Diversos fatores interagem interferindo na motilidade do tubo digestivo e no esvaziamento gástrico ${ }^{(2,5,15,16,23)}$. O tipo de conteúdo ingerido, o volume, a osmolaridade, a acidez, o teor de gorduras, proteínas e carboidratos determinam, por ação mecânica e química, a estimulação de receptores no tubo digestivo. Estes interagem com células neuroendócrinas da mucosa, determinando a secreção de hormônios gastrointestinais, que atuam e fazem a integração entre os múltiplos estímulos do lúmen com aqueles determinados pela enervação intrínseca e extrínseca. Um bom exemplo é o da gastrina que, ao lado do estimulo da secreção

Apoio: JANSSEN-CILAG

$+\quad$ Trabalho realizado no Departamento de Anatomia do ICB/CCS/UFRJ e Serviço de Radiodiagnóstico do HUCFF do CCS da UFRJ

* Médica. Mestre em Medicina.

** Do CCS da UFRJ

Endereço para correspondência: Dra. Claudia M.G. Firman - Laboratório de Motilidade Digestiva/Imagem, Sl. F1-012 - Av. Brigadeiro Trompowiski, s/n - Ilha do Fundão - 21941-590 - Rio de Janeiro, RJ. 
cloridropéptica, é capaz de estimular a contração da musculatura do estômago, acelerando o seu esvaziamento ${ }^{(16)}$.

Diversas patologias como úlcera gástrica ou duodenal, carcinoma do estômago, estenose pilórica, gastroparesia diabética e até taquicardia de origem idiopática têm sido relacionadas como capazes de comprometer a complexa físiologia do esvaziamento gástrico ${ }^{(20)}$. O comprometimento do esvaziamento gástrico não se relaciona necessariamente a um obstáculo mecânico. Com freqüência a disfunção se deve a uma alteração funcional.

As anormalidades no esvaziamento gástrico podem ser decorrentes de doenças do próprio tubo digestivo, de alterações nas conexões desse tubo com o sistema nervoso, de desordens metabólicas ou da musculatura lisa e, ainda, podem ser conseqüência de cirurgias gástricas e distúrbios psicológicos ${ }^{(3,7,9,11)}$.

Do ponto de vista clínico, as desordens do esvaziamento gástrico estão entre as formas mais importantes de dismotilidade do tubo gastrointestinal. Estas alterações podem ser assintomáticas ou estarem relacionadas a sintomas que podem variar de toleráveis até incapacitantes.

O estudo do esvaziamento gástrico se presta a identificar, caracterizar e a estabelecer possíveis correlações fisiopatológicas, mas também é efetiva para testar a eficiência de uma terapêutica medicamentosa ou cirúrgica ${ }^{(10,17,20)}$. O estudo do esvaziamento tem permitido a identificação tanto de trânsito rápido, como lento em pacientes previamente diagnosticados como tendo uma base psicológica para suas queixas digestivas ${ }^{(10)}$.

As diversas técnicas que avaliam o esvaziamento gástrico visam definir, a partir de um conteúdo gástrico inicial de "valor" conhecido, a taxa de eliminação desse conteúdo pelo estômago. Dentre essas técnicas, incluem-se as de intubação-aspiração, a radiológica, a cintilográfica, a ressonância nuclear magnética, a ultra-sonografia, a pletismografia de impedância, a tomografia de impedância, os testes respiratórios, os de absorção de fármacos e o teste do traçador magnético $^{(6,17,18)}$. Apesar da diversidade de técnicas propostas e utilizadas no estudo do esvaziamento gástrico, ainda há muito o que esclarecer na fisiologia desse processo ${ }^{(1,8,12,19,21)}$. As dificuldades de execução e o pouco apuro na interpretação dos resultados, são os fatores apontados como principais responsáveis pela limitação das técnicas ${ }^{(13,14,22)}$.

Um bom método para a avaliação da motilidade e do esvaziamento gástrico deve ser de fácil acesso, reprodutível, baixo custo, de preferência não-invasivo. Deve ser capaz de permitir a visualização da motilidade do antro, do piloro e do duodeno. Deve mostrar o fluxo transpilórico. Deve permitir a caracterização da freqüência e da amplitude das contrações antrais. Sobretudo e evidentemente, ser capaz de permitir a análise da correlação entre a motilidade e o esvaziamento gástrico. Este método parece ser o videofluoroscópico, que é um método de registro radiológico dinâmico que permite a visualização em tempo real da motilidade e do esvaziamento gástrico. A limitação em se quantificar os fenômenos registrados por método radiológico é a barreira a ser vencida ${ }^{(4)}$.

\section{MATERIAL E MÉTODOS}

Avaliou-se o esvaziamento gástrico de 21 voluntários sadios, 14 do sexo feminino e 7 do masculino, com idades compreendidas entre 19 e 39 anos. Os 21 voluntários foram selecionados de um grupo de 33 através de questionário, que excluiu 12 indivíduos que apresentavam sintomas relacionados ao tubo digestivo ou doenças que poderiam interferir nos resultados. Todos os selecionados foram informados do teor da pesquisa e assinaram termo de consentimento.

Os estudos foram efetuados através do método videofluoroscópico utilizando-se seriógrafo, com sistema de TV, com intensificador de imagens e tubo tipo Vidicom, padrão 525 linhas, $60 \mathrm{~Hz}$, e entrelaçamento $2: 1$. As imagens foram gravadas em fita VHS, em videogravador Panasonic J33.

Os voluntários foram examinados em posição ortostática, em incidência póstero-anterior com o écran/intensificador tangente ao seu corpo. Ao écran, fixou-se placa com quadriculado hipotransparente de $2 \mathrm{~cm}$ de lado, que serviu de barra de calibração.

Os voluntários foram examinados com um mínimo de 6 horas de jejum. Utilizaram-se $250 \mathrm{~mL}$ de dieta padrão líquido-pastosa contendo 7,6 g de proteínas (prot.), 31,7 g de glicídios (glic.) e 5,6 g de lipídios (lip.), num total de 210,1 kcal, composta pela homogeneização de:

- $20 \mathrm{~g}$ de leite em pó integral (5,6 g de prot., 7,8 g glic., 4,8 g lip.) $-96.8 \mathrm{kcal}$.

- $10 \mathrm{~g}$ de gelatina (0,6 g prot., $1,8 \mathrm{~g}$ glic.) - 9,9 kcal.

- $10 \mathrm{~g}$ de farinha láctea (1,3 g prot., 7,5 g glic., 0,7 g lip. ) - 42,4 kcal.

- 10 g de açúcar (9,9g glic.) - 39,8 kcal.

- $35 \mathrm{~g}$ de maçã (0,1 g prot., 4,7 g glic., 0,1 g lip.) - 21,2 kcal.

- $50 \mathrm{~mL}$ de água.

- $150 \mathrm{~mL}$ de solução de sulfato de bário (gel comercial a 100\%)

Após ingestão da dieta teste (V0), foram efetuados registros videofluoroscópicos, com um minuto de duração, em intervalos de 12 minutos, num total de seis registros (V0,V1,..V5). Registros complementares, a cada 30 minutos em média após V5, foram efetuadas até a observação do esvaziamento total do estômago.

As seqüências registradas em fita VHS foram analisadas qualitativamente quanto: à motilidade do antro, piloro e duodeno, ao fluxo transpilórico, e à freqüência e amplitude das contrações antrais.

Foram selecionados oito exames, por qualidade de imagem, para serem submetidos a quantificação. Esta foi realizada através de rotina computacional de macros, desenvolvido para este fim, no software Image pro-plus da Media Cibernetycs. 
Foram digitalizadas imagens representativas de cada tempo de V0 a V5. As imagens contendo barra com valores conhecidos, permitiram calibração métrica do sistema de mensuração computacional. Além da calibração métrica, efetuou-se a calibração de densidade, utilizando escala de cinza ( 0 a 255). As calibrações foram registradas na memória do sistema como padrão e utilizadas para todos os tempos obtidos por um mesmo regime.

A área gástrica contrastada foi delimitada e dividida em duas porções, corpo-fundo e antro. A delimitação do antro foi feita traçando-se perpendicular da "incisura angularis" em direção à grande curvatura, formando um ângulo de 45 graus com o plano transverso do corpo gástrico (definição de ângulo permitida pelo software).

As imagens delimitadas e divididas de cada um dos tempos foram mensuradas. As mensurações realizadas geraram valores numéricos em centímetros para as áreas. As suas densidades relativas foram expressas por módulos que representam valores da escala de cinza em que 255 representa hipotransparência máxima e geram valores sem unidade definida.

A partir dos valores de cada tempo registrado, foi possível definir as variações de quantidade da dieta contrastada de V0 a V5 (esvaziamento gástrico).

A rotina computacional desenvolvida no Image-Pro plus, relacionou área e densidade das imagens de cada tempo através de rotina determinada por macros capazes de gerar valores que permitem definir curva de esvaziamento.

Com a imagem (V0) de um determinado exame na tela, a macro 1 determina a criação de um arquivo. A macro 2, carrega a calibração previamente elaborada e define as variáveis a serem mensuradas (área e densidade). Efetua essa mensuração, gera o produto área $\mathrm{x}$ densidade (índice volumétrico de antro e de fundo/corpo) e salva os valores obtidos no arquivo definido na macro 1 ; retira a imagem já trabalhada da tela e solicita a imagem seguinte. Para cada uma das imagens seguintes, a macro 2 é acionada, produzindo seqüência semelhante à gerada para V0.

Com todas as imagens mensuradas e salvas, a macro 3 opera o arquivo de registro, gerando o índice volumétrico corrigido de cada um dos tempos. O índice volumétrico corrigido de cada tempo (IVc) é obtido, dividindo-se o índice volumétrico do antro (IVa) de cada tempo (v1, v2, etc.) pelo índice volumétrico do antro da imagem v0 (IVa/ IVv0 ), multiplicado o resultado pelo índice volumétrico do fundocorpo (IVfc) desse mesmo tempo (v1, v2, etc..).

Os cálculos descritos podem ser expressos na fórmula abaixo:

$\mathrm{IVc}=\mathrm{IVa} . \mathrm{IVfc}$

IVv0

Os dados numéricos obtidos da imagem de cada tempo foram utilizados em "software" de planilha (Excel), para geração de um gráfico (curva de esvaziamento gástrico).

\section{RESULTADOS}

Os voluntários não tiveram dificuldades em aceitar a dieta baritada. O paladar, a consistência e o volume foram considerados adequados aos objetivos.

A utilização de placa fixada ao écran mostrou-se eficaz. Pudemos identificar malha quadriculada sobreposta à imagem gástrica e utilizála como barra de calibração.

As imagens videofluoroscópicas, registradas em fitas VHS, permitem observar a repleção e a seqüência dinâmica do esvaziamento gástrico. Pode-se observar a dinâmica contrátil do estômago, identificando com nitidez o ponto de início da contração, a migração da onda de contração pelo corpo e antro, a abertura pilórica e o fluxo transpilórico.

O tempo de esvaziamento gástrico, registrado nos 21 voluntários estudados por videofluoroscopia, esteve compreendido entre $1: 45 \mathrm{~h}$ e $4 \mathrm{~h}$, com tempo médio de 3:9 h e moda de 2:30 h (Tabela 1).

TABELA 1 - Distribuição dos voluntários quanto ao sexo, idade e o tempo necessário para o completo esvaziamento gástrico medido em horas

\begin{tabular}{rlccc}
\hline $\mathbf{N}^{\mathbf{0}}$ & Ident. & Sexo & Idade & Temp. esv. (h) \\
\hline 1 & R.J.N. & $\mathrm{M}$ & 28 & $2: 20$ \\
2 & C.M.G. & $\mathrm{F}$ & 23 & $2: 40$ \\
3 & L.C.F. & $\mathrm{F}$ & 27 & $2: 26$ \\
4 & L.C.F. & $\mathrm{F}$ & 26 & $4: 00$ \\
5 & R.M.N. & $\mathrm{M}$ & 26 & $2: 57$ \\
6 & L.C.L.S. & $\mathrm{M}$ & 29 & $2: 45$ \\
7 & D.P.G. & $\mathrm{F}$ & 27 & $3: 00$ \\
8 & M.V.T.F. & $\mathrm{F}$ & 21 & $2: 15$ \\
9 & F.I.G. & $\mathrm{M}$ & 19 & $1: 45$ \\
10 & M.P.V. & $\mathrm{F}$ & 30 & $3: 00$ \\
11 & M.F.P. & $\mathrm{F}$ & 26 & $2: 30$ \\
12 & P.M.F.L.D. & $\mathrm{M}$ & 27 & $1: 45$ \\
13 & J.A.S.P. & $\mathrm{F}$ & 34 & $2: 30$ \\
14 & C.E.S.S. & $\mathrm{M}$ & 24 & $3: 30$ \\
15 & I.T.B. & $\mathrm{F}$ & 22 & $3: 15$ \\
16 & M.T.C. & $\mathrm{F}$ & 39 & $3: 10$ \\
17 & A.A.S.R. & M & 23 & $2: 00$ \\
18 & L.M.C.S. & F & 21 & $3: 45$ \\
19 & F.B.D. & F & 19 & $2: 30$ \\
20 & M.T.S.C. & F & 20 & $3: 30$ \\
21 & A.T.P.C. & F & 31 & $2: 30$ \\
\hline
\end{tabular}

Ident. = identificação representado pelas iniciais do voluntário e número do exame; sexo onde $\mathrm{M}=$ masculino, $\mathrm{F}=$ feminino. A idade é referida em anos completos. Temp. esv. = tempo necessário para o completo esvaziamento gástrico de cada voluntário medido em horas 
Não se observaram diferenças significativas quanto ao tempo de esvaziamento gástrico e o comportamento dinâmico do estômago que pudessem ser correlacionadas com sexo e idade dos voluntários.

Em 19 dos 21 voluntários (90\%), as imagens registradas no momento V1 (segundo registro da seqüência) exibiram maior volume do que o observado em V0. Uma faixa hipotransparente de menor densidade que a da dieta se mostrou presente no limite superior dessas imagens. Nesses voluntários um esvaziamento efetivo passou a se processar, a partir de V2. Em dois voluntários (10\%) não foi observado aumento de volume do estômago, mostrando um V1 menor que V0. Um efetivo e seqüencial esvaziamento já pode ser observado a partir de V0.

Em todos os voluntários pudemos observar, após o início do efetivo esvaziamento, que o conteúdo gástrico prossegue sendo eliminado para o delgado de forma gradual e contínua.
O registro da primeira hora do esvaziamento do estômago, de cada um dos voluntários (V0 a V5), permitiu a observação de uma seqüência temporal representativa do esvaziamento gástrico. A partir de V1 (10\%) ou V2 (90\%) pode ser observado um esvaziamento gradual e contínuo. Um efetivo esvaziamento já pode ser definido em V5, onde um resíduo basicamente antral configura uma dinâmica adequada de esvaziamento do estômago. A seqüência de imagens registradas de V0 a V5 mostrou-se capaz de demonstrar, de modo qualitativo, o processo de esvaziamento do estômago.

O processamento matemático das imagens, efetuado pelo programa computacional desenvolvido, permitiu a geração de valores numéricos denominados de índices volumétricos para cada registro de V0 a V5.

As seqüências de imagens digitalizadas e utilizadas para quantificação do esvaziamento também podem ser apresentadas como seqüência qualitativa dos tempos do esvaziamento gástrico, sob a forma de galeria de imagens (Figura 1).

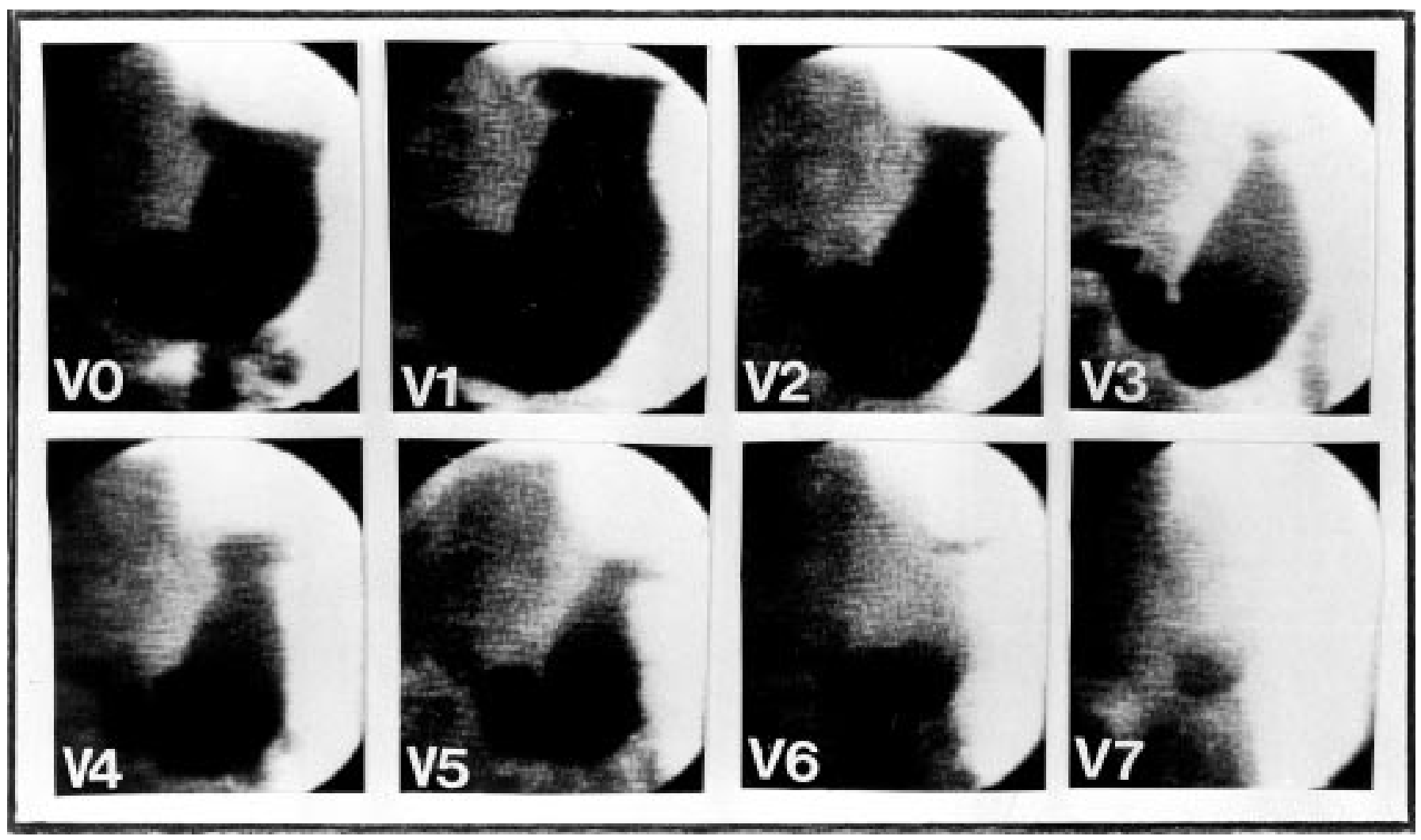

FIGURA 1 - Montagem fotográfica obtida de seqüência de imagens digitalizadas de um dos voluntário onde de V0 a V5 representa a primeira hora e V6 e v7 a hora subseqüente. 
A metodologia de quantificação computacional desenvolvida, permitiu informar, para cada exame analisado, o número de tempos (registros videofluoroscópicos), o número de medidas realizadas em cada tempo, os resultados dessas medidas (área e densidade) das regiões delimitadas

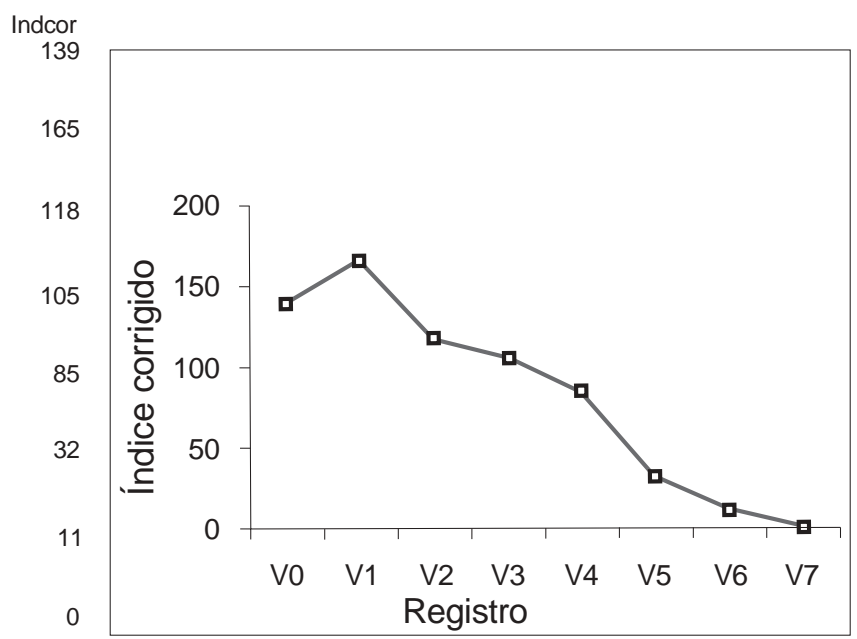

FIGURA 2 - Gráfico demonstrativo de quantificação do esvazia mento gástrico de voluntário. (fundo/corpo e antro), o índice volumétrico de cada uma dessas regiões e um índice corrigido (índice de esvaziamento gástrico corrigido). Estes índices de esvaziamento gástrico corrigidos são fornecidos sob a forma de tabelas e gráficos associados (Figura 2).

Pelo fato de se terem medido as variações do esvaziamento gástrico relacionadas à área e não ao volume, os cálculos foram refeitos seguindo a mesma metodologia, usando as áreas obtidas em lugar do volume. Esses resultados foram submetidos a tratamento estatístico (Tabela 2 e Figura 3 ).

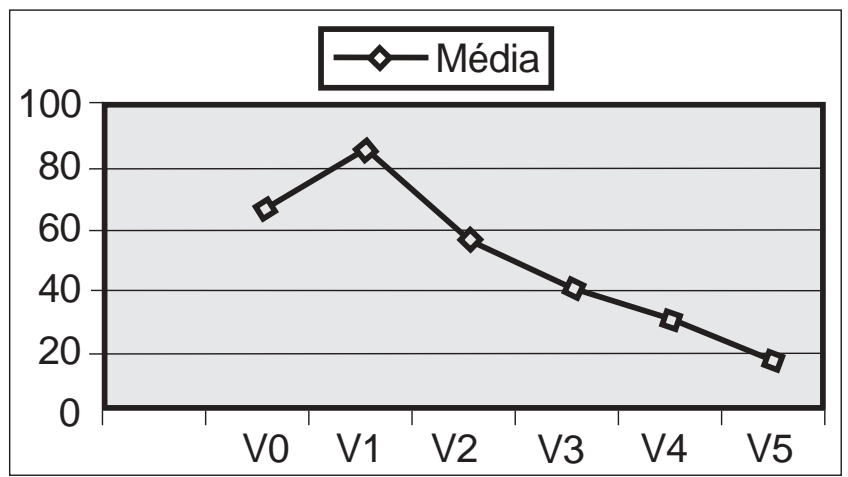

FIGURA 3 - Gráfico obtido plotando-se as médias dos registros.

TABELA 2 - Áreas normatizadas dos registros de V0 a V5 dos oito exames quantificados; resultados da estatística descritiva de cada grupo de registro

\begin{tabular}{|c|c|c|c|c|c|c|c|}
\hline \multicolumn{2}{|l|}{ Média } & 66,7375 & 86,2375 & 56,1875 & 40,7875 & 30,4875 & 17,05 \\
\hline \multicolumn{2}{|l|}{ Erro padrão } & 6,302662 & 10,93537 & 7,231069 & 5,129830 & 5,843416 & 3,417131 \\
\hline \multicolumn{2}{|l|}{ Mediana } & 68,3 & 83,55 & 47,65 & 45,1 & 30,25 & 18,25 \\
\hline \multicolumn{2}{|c|}{ Desvio padrão } & 17,82662 & 30,92991 & 20,45255 & 14,50935 & 16,52767 & 9,665106 \\
\hline \multicolumn{2}{|c|}{ Variância da amostra } & 317,7883 & 956,6598 & 418,3069 & 210,5212 & 273,1641 & 93,41428 \\
\hline \multicolumn{2}{|c|}{ Assimetria } & 0,278235 & 0,339219 & 0,907530 & $-0,737162$ & $-0,001573$ & $-0,653821$ \\
\hline \multicolumn{2}{|l|}{ Intervalo } & 57,7 & 91,2 & 50,4 & 38,9 & 52,5 & 27,1 \\
\hline \multicolumn{2}{|l|}{ Mínimo } & 40,9 & 44,2 & 39 & 18,5 & 4,5 & 2,2 \\
\hline \multicolumn{2}{|l|}{ Máximo } & 98,6 & 135,4 & 89,4 & 57,4 & 57 & 29,3 \\
\hline \multicolumn{2}{|l|}{ Soma } & 533,9 & 689,9 & 449,5 & 326,3 & 243,9 & 136,4 \\
\hline \multicolumn{2}{|l|}{ Contagem } & 8 & 8 & 8 & 8 & 8 & 8 \\
\hline \multirow[t]{2}{*}{ Voluntário } & tempo & área & área & área & área & área & área \\
\hline & $\mathrm{T} 1 / 2$ & V0 & $\mathrm{V} 1$ & V2 & V3 & V4 & V5 \\
\hline 12 & $1 \mathrm{~h}$ & 47 & 119 & 52,6 & 49 & 31,9 & 19,7 \\
\hline 13 & $1 \mathrm{~h}$ & 40,9 & 44,2 & 39 & 18,5 & 13,9 & 3,7 \\
\hline 14 & $1 \mathrm{~h}$ & 70 & 79,3 & 62,9 & 48 & 57 & 16,7 \\
\hline 17 & $1 \mathrm{~h}$ & 62 & 59,6 & 40 & 20,6 & 4,5 & 2,2 \\
\hline 18 & $1 \mathrm{~h}$ & 66,6 & 87,8 & 42,7 & 42,2 & 28,6 & 16,8 \\
\hline 19 & $1 \mathrm{~h}$ & 98,6 & 135,4 & 89,4 & 57,4 & 45,5 & 29,3 \\
\hline 20 & $1 \mathrm{~h}$ & 73,5 & 100,3 & 83,5 & 53,4 & 27,6 & 23,2 \\
\hline 21 & $1 \mathrm{~h}$ & 75,3 & 64,3 & 39,4 & 37,2 & 34,9 & 24,8 \\
\hline Média & & 66,73 & 86,23 & 56,187 & 40,78 & 30,48 & 17,05 \\
\hline Erro padrão & & 6,302 & 10,93 & 7,2310 & 5,129 & 5,843 & 3,417 \\
\hline Mediana & & 68,3 & 83,55 & 47,65 & 45,1 & 30,25 & 18,25 \\
\hline $\begin{array}{l}\text { Desvio } \\
\text { padrão }\end{array}$ & & 17,82 & 30,92 & 20,452 & 14,50 & 16,52 & 9,665 \\
\hline Soma & & 533,9 & 689,9 & 449,5 & 326,3 & 243,9 & 136,4 \\
\hline Contagem & & 8 & 8 & 8 & 8 & 8 & 8 \\
\hline
\end{tabular}




\section{DISCUSS̃̃O}

O uso de uma refeição pastosa com lipídios, carboidratos e proteínas, nas proporções aproximadas de uma refeição normal, misturada à solução de sulfato de bário como meio de contraste, possibilitou a avaliação da motilidade e do esvaziamento do estômago. A homogeneização dos componentes desta dieta com a solução de sulfato de bário, permitiu uma mistura capaz de incorporar este meio de contraste aos demais componentes da dieta, solucionando a dificuldade que era apontada como importante fator limitante para avaliação radiológica do esvaziamento gástrico. Além disso, por já ser pastosa, esta dieta estaria próxima à consistência do quimo a ser ejetado pelo estômago; teoricamente, constitui-se em dieta capaz de representar por média, a dieta sólida e a líquida, configurando-se em mistura adequada para aferição do esvaziamento gástrico $^{(4)}$.

O relaxamento receptivo, a contratilidade coordenada que integra antro, piloro e duodeno ${ }^{(5,11,16)}$, bem como o comportamento peristáltico do período pós-prandial ${ }^{(11)}$ podem efetivamente ser observados.

As imagens videofluoroscópicas permitem analisar a repleção e a seqüência dinâmica do esvaziamento gástrico. Mostram com clareza a dinâmica contrátil do estômago. Permitem identificar com nitidez o ponto de início da contração, a migração da onda de contração pelo corpo e antro, a abertura pilórica e o fluxo transpilórico. Fácil metodização, baixo custo e reprodutibilidade garantem à avaliação videofluoroscópica do estômago, o atendimento dos requisitos básicos para que se possa considerá-lo com um bom método para o estudo da dinâmica e do esvaziamento gástrico.

A admissão de que, apesar dos diversos métodos utilizados ainda há muito o que esclarecer na fisiologia do esvaziamento gástrico ${ }^{(1,9,12}$, 19,21), dá a visão direta e em tempo real permitida pela videofluoroscopia uma vantagem promissora. A definição de um período da "lag fase" apontado pelo método cintilográfico esbarra na observação de $90 \%$ dos indivíduos observados pela videofluoroscopia apresentarem um aumento do volume gástrico neste mesmo período. É possível que estejamos registrando o aporte cloridropéptico. Como não há eliminação do conteúdo gástrico neste momento, a radiação captada pela cintilografia continua limitada ao estômago, sem registrar a existência da dinâmica de aporte de volume como observado pela videofluoroscopia.

O tempo de esvaziamento observado em nossa amostra teve como moda $2: 30 \mathrm{~h}$ dentro dos limites de tempo aceito como nível de normalidade. Cerca de $50 \%$ dos voluntários apresentaram tempo de esvaziamento próximo desta moda, embora a média deste tempo tenha sido de 3:09 h desviada por quatro exames (20\%) que apresentaramno igual ou superior a 3:30 h. É possível que a solução de sulfato de bário tenha, de algum modo, influenciado neste tempo tendente ao retardo observado em nossa amostra. É de se crer que padronizado o método, este não seja um óbice relevante, superado que seria pelo ganho da observação qualitativa permitida pelo registro dinâmico do processo de esvaziamento.

Com o método videofluoroscópico, à semelhança do observado para o método cintilográfico, um efetivo esvaziamento do estômago já pode ser definido na análise da primeira hora (T1/2). Pôde-se observar, após o inicio do efetivo esvaziamento, que o conteúdo gástrico prossegue sendo eliminado para o delgado de forma gradual e contínua. Um efetivo esvaziamento já pode ser definido em V5 onde um resíduo, basicamente antral, configura uma dinâmica adequada. No entanto é conveniente observar que a contração gástrica, a medida que propele o conteúdo no sentido pilórico, por resistência parcial desta estrutura, gera distensão retrógrada do corpo gástrico que, eventualmente, faz parecer existir um maior volume em V5 que em V4. Deste modo, a análise qualitativa importa para compreensão e permite a seleção do melhor momento dentro de cada tempo para a captura das imagens a serem mensuradas como representativas daquele determinado tempo.

O processamento matemático das imagens observadas na seqüência de registros que gerou valores numéricos, denominados de índices volumétricos, teve a pretensão de traduzir através de módulo numérico o esvaziamento gradual e contínuo do volume contido no estômago. Estes índices exprimem o produto de área $\mathrm{x}$ densidade. A densidade substituiria o diâmetro ântero-posterior, cuja mensuração em escala métrica se mostrou impraticável.

Avaliar a variação ântero-posterior mostrou-se importante para que pudéssemos considerar o esvaziamento do estômago em uma fração de seu tempo total (T1/2 - primeira hora do esvaziamento). Ao lado do real esvaziamento gradativo do estômago existe, algumas vezes, um aparente esvaziamento dependente das contrações do órgão. Essas aparentes variações de volume intragástrico, que podem ser percebidas pela avaliação qualitativa e que são dependentes das contrações gástricas com acomodação de volume, são as responsáveis pelas aparentes diminuições de diâmetro e área. Assim, estimar o volume do conteúdo de uma víscera oca, cuja morfologia varia de modo dinâmico com freqüência e intensidade significativas, a partir da mensuração de diâmetros ou áreas, certamente pode nos conduzir a erros de avaliação.

O método de mensuração por nós proposto, ao dividir o índice volumétrico do antro (Iva) de cada tempo pelo índice volumétrico do antro da imagem V0 (IVv0), normatiza e redistribui o volume intragástrico, neutralizando o efeito das variações morfológicas. Esta normatização permite quantificar adequadamente, a cada tempo, o que efetivamente foi esvaziado para o intestino e o que ainda é conteúdo gástrico.

Com esta crença, efetuaram-se as operações que permitiram a obtenção de índices volumétricos corrigidos para cada tempo e, deste modo, pudemos traçar uma curva de esvaziamento para cada caso. 
Apesar dos cuidados, um erro metodológico foi cometido e somente percebido ao final de nossas observações, quando da revisão dos resultados. O regime radiológico (kilovoltagem/miliamperagem) adequado à avaliação da dinâmica gástrica, não permitiu, com o uso da solução de sulfato de bário a $100 \%$, que se quantificasse, através de análise computacional, as variações da densidade nas áreas contrastadas, apesar da evidente redução de volume e densidade que se pode perceber pela análise qualitativa. A radiação, relativamente baixa durante o exame, quantifica com mesmo valor de densidade, níveis de hipotranparência que deveriam ser distintos pela eliminação de volume. Em analise qualitativa vê-se o contraste ser transferido para o intestino. Volume e densidade intragástrica diminuem. No entanto o sistema computacional não tem sensibilidade suficiente para perceber a variação da densidade. Mesmo com um menor volume, a hipotransparência percebida pelo sistema tem valor semelhante ao determinado pelo volume inicial. Assim, na realidade não fomos capazes de perceber as variações de uma terceira dimensão representada pela densidade. As variações que registramos deveram-se a alterações de área. Nossos resultados, de qualquer modo, constituem-se em um índice que registra a dinâmica do esvaziamento gástrico, de modo semelhante a tantos outros que até aqui têm avaliado este esvaziamento.

Como contraprova e com a finalidade de demostrar similaridade entre os resultados obtidos e aqueles que se obteria usando-se somente a medida de área, os cálculos foram refeitos com a mesma metodologia, desprezando-se as densidades aferidas.

Os resultados obtidos foram agrupados segundo os tempos de registro de V0 a V5 e submetidos a tratamento estatístico descritivo. Os resultados expressos em área $\left(\mathrm{cm}^{2}\right)$ se superpuseram, caso a caso, àqueles nos quais consideramos as densidades. As médias, medianas e somas dos diversos tempos configuram curva que reproduz o padrão que correspondeu a $90 \%$ de nossos achados, conforme análise da Tabela 2.

Nossos resultados, aferindo área, expressaram uma consistente seqüência capaz de representar o padrão de esvaziamento do estômago, como tanto outros métodos. No entanto, as variações no diâmetro ântero-posterior continuam sem ser aferidas, podendo falsear resultados. Um verdadeiro e confiável índice volumétrico só será obtido quando a quantificação deste índice considerar as três dimensões do estômago. Diluições do meio de contraste devem ser experimentadas para que se possa conseguir as variações de uma terceira dimensão, o que, efetivamente, nos permitiria falar em um índice volumétrico para o esvaziamento gástrico.

\section{CONCLUSÕES}

A pesquisa realizada autoriza-nos a concluir que:

1. A videofluoroscopia permite, com adequada resolução, a análise qualitativa da motilidade e do esvaziamento gástrico.

2. É possível se quantificar as variações de forma observáveis pelos métodos radiológicos de registro seqüencial de imagens, mormente as geradas pelo método videofluoroscópico que registra 30 frames por segundo.

3. Os nossos resultados expressam um índice de esvaziamento gástrico, determinado pela variação da área de projeção gástrica, em imagens radiológicas obtidas em incidência póstero-anterior.

4. A análise quantitativa não deve prescindir da análise qualitativa.

Firman CMG, Costa MMB, Costa ML, Lemme E. Qualitative and quantitative analysis of the gastric emptying by the videofluoroscopic method. Arq Gastroenterol 2000 37(2):81-88.

ABSTRACT - We studied the gastric emptying of 21 healthy volunteers using a standard contrasted diet composed of a lipid, carbohydrate, protein and barium sulfate solution homogenate. We did six one-minute videofluoroscopic recordings in a 12-minute interval for the first hour, and in a 30 minute interval until the complete emptying. We selected eight exams for the emptying quantification. We could observe and estimate subjectively the functional behavior and the gastric emptying. Digital representative images of each emptying time point we were able to quantify those observations and describe them as a mathematical function. We developed a macro procedure using the Media Cybernetics ImagePro-Plus software for this quantification. We conclude that: 1 - The videofluoroscopic method provides better resolution than the other methods in the analysis of the gastric motility and emptying. 2 - It is possible to computationally quantify the morphological differences observed with the sequential registration radiological methods, specially the videofluoroscopic method that generates 30 frames per second. 3 - Our results generate a gastric emptying index, based on the area of a gastric projection in radiological images obtained in a standing position over a one-hour period (T1/2). 4- The quantitative analysis should not exclude the qualitative analysis.

HEADINGS - Fluoroscopy. Gastric motility. Gastric emptying. 


\section{REFERÊNCIAS BIBLIOGRÁFICAS}

1. Berstad A, Hausken T, Gilja OH, Thune N, Matre K, Odegaard S. Volume measurements of gastric antrum by 3-D ultrasonography and flow measurements through the pylorus by duplex technique. Dig Dis Sci 1994;39:97-100.

2. Brand SJ, Schmidt WE. Gastrointestinal hormones. In: Yamada T, Alpers DH, Owyang C, editors. Textbook of Gastroenterology. 2. ed. Philadelphia: J. B. Lippicott; 1995. p.25-71.

3. Chaudhuri TK, Fink S. Gastric emptying in human disease states. Am J Gastroenterol 1991;86:533-8.

4. Firman CMG. Avaliação e quantificação do esvaziamento gástrico através do método videofluoroscópico. [Dissertação]. Rio de Janeiro: Faculdade de Medicina da Universidade Federal do Rio de Janeiro; 1997.

5. Hasler WL. The physiology of gastric motility and gastric emptying. In: Yamada T, Alpers DH, Owyang C, editors. Textbook of Gastroenterology. 2.ed. Philadelphia: J B Lippicott; 1995. p.181-206.

6. Horowitz M, Dent J, Fraser R, Sun W, Hebbard G. Role and integration of mechanisms controlling gastric emptying. Dig Dis Sci 1994;39:24-7.

7. Lin HC, Hasler WL. Disorders of gastric emptying. In: Yamada T, Alpers DH, Owyang C, editors. Textbook of Gastroenterology. 2.ed. Philadelphia: J B Lippicott; 1995. p.1318-46.

8. Malagelada J-R, Stanghellini V. Manometric evaluation of functional upper gut symptoms. Gastroenterology 1985;88:1223-31.

9. Malagelada J-R. Conscious perception of gut activity. Dig Dis Sci 1994;39:151-3.

10. Malmuds LS, Vitti RA. Gastric emptying. J Nucl Med 1990;31:1499-500.

11. McCallum RW. Motor function of the stomach in health and disease. In: Sleisenger MH, Fordtran JS, editors. Gastrointestinal disease: pathophysiology, diagnosis, management. 4.ed. Philadelphia: W. B. Saunders; 1989. p.675- 713.

12. Parkman HP, Harris AD, Krevsky B, Urbain JL, Maurer AH, Fisher RS. Gastroduodenal motility and dysmotility: an update on techniques available for evaluation. Am J Gastroenterol 1995;90:869-92.
13. Parkman HP, Miller MA, Fisher RS. Role of nuclear medicine in evaluation of patients with suspected gastrointestinal motility disorders. Sem Nucl Med 1995;25:289-305

14. Perkel MS, Fajman WA, Hersh T, Moore C, Davidson ED, Haun C. Comparison of the barium test meal and the gamma camera scanning technic in measuring gastric emptying. South Med J 1981;74:1065-8.

15. Raybould HE, Zittel TT, Holzer HH, Lloyd KC, Meyer JH. Gastroduodenal sensory mechanisms and CCK inibition of gastric emptying response to meal. Dig Dis Sci 1994;39(12):41-3.

16. Read NW, Houghton LA. Fisiologia do esvaziamento gástrico e fisiopatologia da gastroparesia. Clín Gastroenterol Am Norte 1989;2:393-410.

17. Rezende-Filho J. Esvaziamento gástrico: métodos de avaliação. In: Castro LP, Savassi-Rocha PR, Cunha-Melo JR, editores. Tópicos em gastroenterologia 5. Rio de Janeiro: Medsi; 1994. p. 35-47.

18. Rezende-Filho J. Métodos de avaliação da motilidade gástrica. In: Resumos do II Simpósio de Motilidade Digestiva, São Paulo; 1996. p.11-4.

19. Schiwizer W, Fraser R, Fried M. Measurement of gastric emptying and gastric motility by magnetic resonance imaging. Motility 1996;33:8-11.

20. Chang TM, Passaro EP Jr, Su DJ, Hwang CC, Law SL, Pai SH, Chen W. Technectium 99m- dtpa microcapsules: a new preparation for gastric emptying studies. Am J Surg 1986;151:722-4.

21. Urban LJ, Charkes ND. Recent advances in gastric emptying scintigraphy. Sem Nucl Med 1995;25:318-25.

22. Vantrappen G. Methods to study gastric emptying. Dig Dis Sci 1994;39(12):91-4.

23. Weisbrodt NW. Basic control mechanisms. In: Akermans LMA, Johnson A G, Read N W, editors. Gastric and gastroduodenal motility. New York: Praeger; 1984. p.3-20. 